\title{
Real-space Green's tensors for stress and strain in crystals with cubic anisotropy
}

\author{
David A. Faux ${ }^{\text {a) }}$ and Ursula M. E. Christmas \\ Advanced Technology Institute, University of Surrey, Guildford, GU2 7XH, United Kingdom
}

(Received 28 October 2004; accepted 6 June 2005; published online 12 August 2005)

\begin{abstract}
Real-space Green's tensors are presented for the calculation of the stress or strain in infinite cubic crystals containing buried quantum wires or dots of arbitrary shape and composition, including the cubic anisotropy of elastic constants. The Green's tensors are obtained as a polynomial series to second order in $\Delta$, an expansion coefficient defined in terms of the elastic stiffnesses. The zeroth-order term in the series is the usual isotropic Green's tensor. The results agree extremely well with the numerical, exact formulation of Pan and Yang [E. Pan and B. Yang, J. Appl. Phys. 90, 6190 (2001)] but compute considerably faster and are easier to implement. The present approach is used to determine the strain in the direction normal to the plane of a quantum well in different orientations. This constitutes a stringent test of the approximate Green's tensor series and results are found to be in excellent agreement with standard solutions. The Green's-function expansion for the hydrostatic strain is presented and found to be of a simple form for both dots and wires. (c) 2005 American Institute of Physics. [DOI: 10.1063/1.1990266]
\end{abstract}

\section{INTRODUCTION}

Semiconductor quantum dot (QD) and quantum wire (QWI) nanostructures have generated considerable experimental and theoretical interest during the last ten years because of the physics associated with the quantum confinement of carriers to less than three dimensions. Devices offer the realistic prospect of improved electronic and optical properties arising from the reduced dimensionality. QD lasers, for example, offer the possibility of reduced threshold currents, reduced temperature sensitivity, and increased differential gain allowing higher-speed operation at reduced power. Improved devices based on QD or QWI nanostructures can be engineered with desirable properties by the control of the materials, band gap, strain, size, shape, and orientation. ${ }^{1,2}$

Strain is almost always present in QD or QWI systems because of the use of different materials with different lattice constants. The strain distribution in and around individual quantum nanostructures has a strong influence on the electronic and optical properties of devices, on the transmission electron microscopy (TEM) image contrasts, and on the mechanics of growth. For example, Tadic et al. demonstrate the effect of isotropic and anisotropic elasticity on the electronic structure of InP-based systems ${ }^{3,4}$ and Heidemeyer et al. demonstrate the importance of strain in the production of vertically aligned QD stacks. ${ }^{5}$ Bimberg et al. discuss the influence of the strain on the electronic and optical properties of quantum heterostructures. $^{2}$

There are several methods available to calculate strain distributions in nanostructures with finite-element, Green'sfunction, Fourier series, and atomistic approaches the most prominent. Each has its strengths and weaknesses. A particular approach may or may not take into account the anisotropy

${ }^{a)}$ Electronic mail: d.faux@surrey.ac.uk of the elastic constants, the use of different elastic constants for different materials, strain-dependent elastic constants, nanostructure/matrix boundary effects, the presence of free surfaces, electromechanical coupling to piezoelectric fields, and nonlinear strain. Whether the neglect of one or more of these influences is significant depends on the properties under examination. Sometimes the simplest isotropic approximation will suffice, sometimes the more subtle details of the strain field are crucial to understand a particular observation.

Strain-evaluation procedures can be divided into two broad types, atomistic and continuum. Atomistic approaches can be extremely useful and the size of systems which can be explored has increased significantly in recent years. ${ }^{6-9}$ Atomistic simulations are limited by the quality of force fields, by the size of the system which can be modeled with a reasonable computational effort, and by periodic boundary conditions. Of the continuum techniques, the finite-element, Fourier, and Green's approaches dominate the literature. The choice of strain-evaluation technique will depend on the problems to be tackled. In general, finite-element approaches are most useful for complex structures such as the quantum dots on a surface ${ }^{3,10}$ and the Fourier technique can be useful for the calculation of electronic properties whereby the explicit calculation of the strain distribution can be bypassed. ${ }^{11}$

In this paper, we focus on the Green's approach to the calculation of stress and strain in and around buried nanostructures. Simply, the Green's tensor gives the response at a field point due to a unit influence at the origin. The displacement, strain, or stress Green's tensor, when integrated over the surface or volume of a QD (or the cross-sectional area or boundary of a QWI) yields the displacement, strain, or stress tensor, respectively, at the chosen field point due to the QD (or QWI). Green's tensor methods are generally quick and portable. Large systems involving many nanostructures with graded composition pose no difficulty and analytic expressions are sometimes tractable to provide deeper insight into 
TABLE I. Elastic parameters for common semiconductor materials (Ref. 43). The anisotropy coefficient is equal to $\left(C_{11}-C_{12}\right) / 2 C_{44} . \Delta$ and $r$ are expansion coefficients defined by Eqs. (12) and (10), respectively.

\begin{tabular}{rrrrcrr}
\hline \hline & $\begin{array}{c}C_{11} \\
(\mathrm{GPa})\end{array}$ & $\begin{array}{c}C_{12}=\lambda \\
(\mathrm{GPa})\end{array}$ & $\begin{array}{c}C_{44}=\mu \\
(\mathrm{GPa})\end{array}$ & $\begin{array}{c}\text { Anisotropy } \\
\text { coefficient }\end{array}$ & $r$ & $\Delta$ \\
\hline $\mathrm{Si}$ & 165.77 & 63.93 & 79.62 & 0.64 & 0.29 & -0.26 \\
$\mathrm{Ge}$ & 124.00 & 41.30 & 68.30 & 0.61 & 0.30 & -0.30 \\
$\mathrm{AlP}$ & 165.00 & 75.50 & 39.70 & 1.13 & 0.13 & 0.07 \\
$\mathrm{AlAs}$ & 120.20 & 57.00 & 58.90 & 0.54 & 0.38 & -0.31 \\
$\mathrm{AlSb}$ & 87.69 & 43.41 & 40.76 & 0.54 & 0.38 & -0.30 \\
$\mathrm{GaP}$ & 140.50 & 62.03 & 70.33 & 0.56 & 0.36 & -0.31 \\
$\mathrm{GaAs}$ & 118.80 & 53.80 & 59.40 & 0.55 & 0.36 & -0.31 \\
$\mathrm{GaSb}$ & 88.34 & 40.23 & 43.22 & 0.56 & 0.36 & -0.30 \\
$\mathrm{InP}$ & 101.10 & 56.10 & 45.60 & 0.49 & 0.43 & -0.31 \\
$\mathrm{InAs}$ & 83.29 & 45.26 & 39.59 & 0.48 & 0.43 & -0.33 \\
$\mathrm{InSb}$ & 66.69 & 36.45 & 30.20 & 0.50 & 0.42 & -0.31 \\
\hline \hline
\end{tabular}

the physics, revealing trends and simple relations. ${ }^{12-20}$ For example, within the isotropic approximation, full analytical expressions for the strain distribution have been derived for ellipsoidal, cuboidal, and truncated-pyramidal dots ${ }^{13,16}$ and for quantum wires of arbitrary shape ${ }^{12}$ and near to free surfaces. ${ }^{15}$ The Green's technique is a powerful method for the calculation of QD- or QWI-induced strain distributions and its ease of application makes it attractive to practitioners in the field.

Most applications of the Green's approach assume isotropy of the elastic constants. For some problems an isotropic solution may be justified because there are much greater uncertainties (such as dot size and shape) which dominate the accuracy of the calculation, or anisotropy is not significant for the phenomena under investigation. Yet most semiconductors are cubic crystals with an anisotropy coefficient [defined in terms of the elastic constants by $\left.\left(C_{11}-C_{12}\right) / 2 C_{44}\right]$ typically equal to 0.5 compared to the isotropic value of 1 (see Table I), suggesting that the simple isotropic approach may miss some key features of the strain field. For example, Holý et al. and Pinczolits et al. demonstrated that the lateral ordering of QDs can be explained only if the full cubic anisotropy of the crystal is taken into account, ${ }^{21,22}$ Faux and Pearson demonstrated that the hydrostatic strain (which influences the electronic structure) is finite in the matrix material outside the QD if the anisotropy of the elastic stiffnesses is taken into account but zero if isotropy is assumed, ${ }^{23}$ Pan and Yang demonstrated significant differences between isotropic and anisotropic strain fields for a QD close to a free surface, ${ }^{24}$ and Tadić et al. showed that the calculated values of electron and hole energies are influenced by the anisotropy of the crystal structure. ${ }^{3}$ It is reasonable to assume that, of all the approximations pertinent to a strain evaluation, neglect of the cubic anisotropy of the elastic constants is potentially the most important.

Classic work by Burgers, ${ }^{25}$ who determined some terms of a series approximation for a linear elastic material with cubic symmetry; Barnett, ${ }^{26}$ who determined the elastic Green's functions for cubic crystals that require a single numerical integral, and Mura and Kinoshita, ${ }^{27}$ who determined a series equation for the Green's functions for cubic crystals, all contributed to our knowledge of strain fields in cubic materials many years before semiconductor QDs became important. Notable recent work has been performed by Pan and co-workers who have determined the Green's tensor including the effect of electromechanically coupled piezoelectric fields for a QD or a QWI buried in an anisotropic full or half-space. ${ }^{24,28-30}$ For the case of a QD, Pan's Green's tensor is nearly analytic, requiring the numerical solution to an eighth-order polynomial equation followed by a numerical differentiation to evaluate the stress or strain. However, in most III-V systems piezoelectric fields are not important [IIInitride systems and InAs/GaAs with the (111) orientation are exceptions].

In 2000, ${ }^{13}$ we published simple expressions for the strain Green's tensor that enabled the strain to be calculated in QD systems including the cubic anisotropy of the elastic stiffnesses. The Green's tensor was expressed as a polynomial series with the zeroth-, first-, and second-order terms presented. Although an approximate solution, truncation at second order appeared to provide a very good approximation to the exact solution for the specific case of a spherical QD.

In this paper we present the theory leading to explicit real-space Green's tensors which enable the stress and strain distributions in and around QDs and QWIs to be calculated rapidly and accurately including the cubic anisotropy of the elastic stiffnesses. The Green's tensor is compared directly to the formalism of Pan and Yang ${ }^{24}$ and we show that the polynomial expression for the Green's tensor is indeed very accurate.

There are many advantages to using the real-space Green's tensor expansion presented here compared to the Pan-Yang formalism. First, the implementation is very straightforward as the determination of either the stress or strain involves a volume integral (surface integral for wires) of a simple polynomial function. The Pan-Yang approach is much more complex to implement as several stages are required to determine the Green's function for displacement followed by numerical differentiation to obtain the strain Green's function, then a surface integral to calculate the strain. The computation of the strain at a field point, for example, is about 50 times faster using the Green's tensor expansion than the Pan-Yang method. One example of a result that can only be computed using the current method, and 
not with the Pan-Yang approach, is the estimation of the energy of interaction of two point defects. ${ }^{31}$ Here the difference of two energies is required, each energy computed by integrating the product of two strains over a large volume of space. The computational resource required for the Pan-Yang approach is too demanding to be practical at present to obtain accurate energies. Even if computation time was not a problem, we found that numerical computations intrinsic to the Pan-Yang approach lead to results that are only accurate to three or four significant figures. Normally, this would be perfectly acceptable, but when two similar energies are subtracted, the numerical errors become significant.

This paper is organized as follows. The theory is presented in Sec. II for both QDs and QWIs, based on the work of Mura and Kinoshita. ${ }^{27}$ Comparison to the Pan-Yang formalism and to exact results in certain limits is presented in Sec. III. We draw our conclusions in Sec. IV.

\section{THEORY}

\section{A. Quantum dot}

The Green's tensor expressions determined in this section are derived from the work of Mura and Kinoshita ${ }^{27}$ who described, from first principles, how the Green's tensor for anisotropic elasticity can be determined in a series form based on the work of Lifshits and Rosentsveig. ${ }^{32}$ Their expressions, which are mathematically complex in the general case, can be simplified for anisotropic cubic crystals. Mura and Kinoshita did not evaluate explicitly the Green's tensors, instead, they provided a series expression involving a large number of differentials with respect to the Cartesian spatial coordinates. Modern computer algebra packages enable the differentials to be executed and the full Green's tensors obtained. Below, the results of Mura and Kinoshita's theory for anisotropic cubic crystals are summarized using, as far as practical, their notation. This theory is then used to produce the Green's tensors for stress and strain for both quantum dots and wires.

The theory that follows is developed for the case of a QD inclusion. It is assumed that the elastic properties are linear, that the same elastic stiffnesses pertain to the QD and the surroundings, and that the QD is embedded in an infinite matrix. The use of the elastic stiffnesses of the matrix material for both matrix and QD is the most significant approximation and this has been discussed elsewhere. ${ }^{13,33,34}$ Currently, even the most sophisticated Green's analyses assume constant elastic stiffnesses for all materials. ${ }^{24,28}$

The calculation begins with the evaluation of a $\mathbf{3} \times \mathbf{3}$ tensor $V_{k, 6 n+5}(\boldsymbol{\omega})$ whose elements are polynomials of degree $6 n+5$ and where $\boldsymbol{\omega}=\left(\omega_{1}, \omega_{2}, \omega_{3}\right)$ is a unit vector. There is one tensor for each spatial direction represented by $k$ $=1,2,3$. The $x_{1}, x_{2}$, and $x_{3}$ axes correspond to the (100), (010), and (001) crystallographic directions, respectively. $V_{k, 6 n+5}(\boldsymbol{\omega})$ is given by

$$
V_{k, 6 n+5}(\boldsymbol{\omega})=\left.|\boldsymbol{\omega}|^{6 n+8} \frac{\partial \hat{G}_{n}(\boldsymbol{\xi})}{\partial \xi_{k}}\right|_{\xi=\boldsymbol{\omega}},
$$

in which $\hat{G}_{n}(\boldsymbol{\xi})$ is a rank 3 tensor and $\boldsymbol{\xi}=\left(\xi_{1}, \xi_{2}, \xi_{3}\right)$ is a vector. The term $|\boldsymbol{\omega}|^{6 n+8}$ is equal to 1 (because $\boldsymbol{\omega}$ is a unit vector) and is included to ensure that this expression remains dimensionally correct and homogeneous of order $6 n+5$ in $|\boldsymbol{\omega}|$. Readers seeking the full mathematical treatment leading to Eq. (1) are referred to Mura and Kinoshita. ${ }^{27}$

For crystals with cubic anisotropy, the function $\hat{G}_{n}(\xi)$ may be written as ${ }^{27}$

$$
\begin{aligned}
& \hat{G}_{n}(\boldsymbol{\xi})=(-1)^{n} \Omega(\boldsymbol{\xi})^{n} \frac{N(\boldsymbol{\xi})}{|\boldsymbol{\xi}|^{6 n+6}}, \\
& \Omega(\boldsymbol{\xi})=b \mid \xi^{2}\left(\xi_{2}^{2} \xi_{3}^{2}+\xi_{3}^{2} \xi_{1}^{2}+\xi_{1}^{2} \xi_{2}^{2}\right)+c \xi_{1}^{2} \xi_{2}^{2} \xi_{3}^{2}, \\
& a=\mu^{2}\left(\lambda+2 \mu+\mu^{\prime}\right), \\
& b=a^{-1} \mu \mu^{\prime}\left(2 \lambda+2 \mu+\mu^{\prime}\right), \\
& c=a^{-1} \mu^{\prime 2}\left(3 \lambda+3 \mu+\mu^{\prime}\right),
\end{aligned}
$$

where $\mu=C_{44}$ and $\lambda=C_{12}$ are the usual Lamé constants and $\mu^{\prime}=C_{11}-C_{12}-2 C_{44}$ is the anisotropy index. Cubic crystals are isotropic when $\mu^{\prime}=0$ in which case $b=c=\Omega=0$ and only the $n=0$ term of Eq. (2) remains. $N(\xi)$ is ${ }^{27}$

$$
N(\boldsymbol{\xi})=\left(\begin{array}{ccccc}
\mu^{2}|\boldsymbol{\xi}|^{4}+\beta|\boldsymbol{\xi}|^{2}\left(\xi_{2}^{2}+\xi_{3}^{2}\right)+\gamma \xi_{2}^{2} \xi_{3}^{2} & -(\lambda+\mu) \xi_{1} \xi_{2}\left(\mu|\xi|^{2}+\mu^{\prime} \xi_{3}^{2}\right) & \cdots & \cdots & -(\lambda+\mu) \xi_{1} \xi_{3}\left(\mu|\xi|^{2}+\mu^{\prime} \xi_{2}^{2}\right) \\
-(\lambda+\mu) \xi_{2} \xi_{1}\left(\mu|\xi|^{2}+\mu^{\prime} \xi_{3}^{2}\right) & \mu^{2}|\boldsymbol{\xi}|^{4}+\beta|\xi|^{2}\left(\xi_{3}^{2}+\xi_{1}^{2}\right)+\gamma \xi_{3}^{2} \xi_{1}^{2} & \cdots & \cdots & -(\lambda+\mu) \xi_{2} \xi_{3}\left(\mu|\xi|^{2}+\mu^{\prime} \xi_{1}^{2}\right) \\
-(\lambda+\mu) \xi_{3} \xi_{1}\left(\mu|\xi|^{2}+\mu^{\prime} \xi_{2}^{2}\right) & -(\lambda+\mu) \xi_{3} \xi_{2}\left(\mu|\xi|^{2}+\mu^{\prime} \xi_{1}^{2}\right) & \cdots & \cdots & \mu^{2}|\boldsymbol{\xi}|^{4}+\left.\beta|\xi|\right|^{2}\left(\xi_{1}^{2}+\xi_{2}^{2}\right)+\gamma \xi_{1}^{2} \xi_{2}^{2}
\end{array}\right)
$$

where

$$
\begin{aligned}
& \beta=\mu\left(\lambda+\mu+\mu^{\prime}\right), \\
& \gamma=\mu^{\prime}\left(2 \lambda+2 \mu+\mu^{\prime}\right) .
\end{aligned}
$$

The expansion of Eq. (2) is valid if $r<1$, where

$$
r=\max (|b / 4|,|b / 3+c / 27|) .
$$

Mura and Kinoshita included a table of some common materials, mainly metals, and showed that typical values of $r$ range from 0.5 to 0.7 . The values of $r$ for common semiconductor compounds are listed in Table I and typically range from 0.3 to 0.45 . Therefore the series expansion will con- 
TABLE II. The coefficients $K_{i j}^{\sigma \odot}$ are of the form $f\left(A \mu^{2}+B \mu \lambda+C \lambda^{2}\right)$ where $\mu$ and $\lambda$ are the Lamé constants.

\begin{tabular}{rrrrrr}
\hline \hline$i$ & $j$ & \multicolumn{1}{c}{$f$} & $A$ & $B$ & $C$ \\
\hline 3 & 0 & 2 & 22 & 41 & 57 \\
2 & 0,1 & -3 & 34 & 97 & 17 \\
1 & 0,2 & 3 & 18 & 49 & 33 \\
& 1 & -18 & 4 & 12 & 9 \\
0 & 0,3 & -1 & 10 & 5 & -3 \\
& 1,2 & -36 & 0 & 0 & 1 \\
\hline \hline
\end{tabular}

verge more rapidly for semiconductor materials than for most metals.

The real-space Green's tensor can be obtained by summation over $k$ and $n$, thus,

$$
G^{\sigma \odot}(\mathbf{x})=-\frac{1}{4 \pi a} \sum_{n=0}^{\infty} \frac{1}{(6 n+6) !} \sum_{k=1}^{3} \frac{x_{k}}{|\mathbf{x}|^{2}} V_{k, 6 n+5}(\partial)|\mathbf{x}|^{6 n+5},
$$

where $\mathbf{x}=\left(x_{1}, x_{2}, x_{3}\right)$ are the usual Cartesian coordinates, $|\mathbf{x}|^{2}=x_{1}^{2}+x_{2}^{2}+x_{3}^{2}$, and where the superscripts " $\sigma$ " and " $\odot$ " have been added to indicate that the Green's tensor is for stress and for the case of QDs, respectively.

In Eq. (11) the components of the unit vector $\boldsymbol{\omega}$ in Eq. (1) have been replaced by the differential operator $\partial$ so that, for instance, a term in the matrix $V_{k, 6 n+5}(\boldsymbol{\omega})$ involving $\boldsymbol{\omega}_{i}^{j}$ is replaced by the differential operator $\partial^{j} / \partial x_{i}$ acting on $|\mathbf{x}|^{6 n+5}$. As terms in the matrix $V_{k, 6 n+5}$ are homogeneous of order $6 n+5$ in $\left(\omega_{1}, \omega_{2}, \omega_{3}\right), V_{k, 6 n+5}$ involves terms in which $\omega_{1}, \omega_{2}$, and $\omega_{3}$ may each be raised to a power between 0 and $6 n$ +5 , but where the sum of the powers is equal to $6 n+5$. This means that $V_{k, 6 n+5}(\partial)$ contains terms with operators of the form $\left(\partial^{i} / \partial x_{1}\right)\left(\partial^{j} / \partial x_{2}\right)\left(\partial^{6 n+5-i-j} / \partial x_{3}\right)$ with each $i$ and $j$ taking values between 0 and $6 n+5$. If we take the $n=2$ term, for example, $|\mathbf{x}|^{6 n+5}$ has to be differentiated a total of 17 times in each of $18 \times 18$ different ways. This would be a formidable task for even the most ardent theorist!

Mura and Kinoshita proceeded no further than Eq. (11) but the calculation can be performed using an algebraic package such as XMAPLE. ${ }^{35}$ The aim is to obtain the stress Green's tensor expansion where the first term is the isotropic stress Green's tensor and subsequent terms are successive orders of correction for the anisotropic case. In order to conveniently express the tensor expansion, a quantity, $\Delta$, is defined where

TABLE III. The coefficients $K_{i j}^{\epsilon \odot}$ are of the form $f\left(A \mu^{2}+B \mu \lambda+C \lambda^{2}\right)$ where $\mu$ and $\lambda$ are the Lamé constants.

\begin{tabular}{rrrrrr}
\hline \hline$i$ & $j$ & $f$ & $A$ & $B$ & $C$ \\
\hline 3 & 0 & 2 & 14 & 19 & 9 \\
2 & 0,1 & -9 & 14 & 35 & 17 \\
1 & 0,2 & 9 & 6 & 19 & 15 \\
& 1 & -36 & 2 & 3 & 0 \\
0 & 0,3 & -1 & 2 & 1 & 9 \\
& 1,2 & 6 & 4 & 12 & 3 \\
\hline \hline
\end{tabular}

TABLE IV. The coefficients $L_{k i}^{\odot}$ are of the form $f\left(A \mu^{2}+B \mu \lambda+C \lambda^{2}\right)$ where $\mu$ and $\lambda$ are the Lamé constants.

\begin{tabular}{rrrrrr}
\hline \hline$k$ & $i$ & $f$ & $A$ & $B$ & $C$ \\
\hline 2 & 0 & 3 & 22 & 51 & 39 \\
1 & 0,1 & -3 & 26 & 83 & 42 \\
0 & 0,2 & 3 & 22 & 41 & 24 \\
& 1 & -3 & 26 & 93 & 57 \\
\hline \hline
\end{tabular}

$$
\Delta=\frac{\mu^{\prime}}{\lambda+2 \mu}
$$

The elements contained in the $n=0,1, \ldots$, tensors of Eq. (11) can be expanded in terms of $\Delta$, provided $|\Delta|<1$, and terms involving increasing powers of $\Delta$ can be collected to yield an expansion of the form

$$
G^{\Xi \odot}=G_{0}^{\Xi \odot}+\Delta G_{1}^{\Xi \odot}+\Delta^{2} G_{2}^{\Xi \odot} \cdots=\sum_{m=0}^{\infty} \Delta^{m} G_{m}^{\Xi \odot} .
$$

This expression applies to both stress and strain, in other words, the superscript $\Xi$ can be replaced by $\epsilon$ to represent strain or by $\sigma$ to represent stress. Equation (13) for stress is equivalent to Eq. (11) where the expansion is expressed in increasing powers of $\Delta$.

It is now convenient to revert to nontensorial Voigt notation as the preferred way to express stress and strain components. In this case, Eq. (13) represents an expansion in terms of six-element column matrices. Using Voigt notation, the strain components are obtained from the stress components in the usual way through Hooke's Law, namely,

$$
G^{\epsilon \odot}=C^{-1} G^{\sigma \odot},
$$

where $C^{-1}$ is the inverse of the matrix

$$
C=\left(\begin{array}{cccccc}
C_{11} & \lambda & \lambda & 0 & 0 & 0 \\
\lambda & C_{11} & \lambda & 0 & 0 & 0 \\
\lambda & \lambda & C_{11} & 0 & 0 & 0 \\
0 & 0 & 0 & 2 \mu & 0 & 0 \\
0 & 0 & 0 & 0 & 2 \mu & 0 \\
0 & 0 & 0 & 0 & 0 & 2 \mu
\end{array}\right)
$$

where we note the presence of $2 \mu$ in the matrix necessary to convert the stresses defined by Mura and Kinoshita into the usual form for the strains. $G_{0}^{\Xi \odot}$ is obtained from the $n=0$ term of Eq. (11) only, $G_{1}^{\Xi \odot}$ is obtained from the $n=0$ and $n=1$ terms, $G_{2}^{\Xi \odot}$ from the $n=0, n=1$, and $n=2$ terms, and so on. If we take the isotropic limit, $\mu^{\prime}=0$, all terms disappear except for the zeroth which therefore contains the wellknown stress or strain Green's tensor for isotropic elasticity. The dimensionless quantity $\Delta$ is presented in Table I for a range of semiconductors and is typically equal to $\sim-1 / 3$.

We have found that the $m$ th Green's six-element matrix of the series, $G_{m}^{\Xi \odot}$, in Eq. (13) may be written in terms of two functions, 
TABLE V. The coefficients $M_{i j}^{\sigma \odot}$ are of the form $f\left(A \mu^{3}+B \mu^{2} \lambda+C \mu \lambda^{2}\right.$ $+D \lambda^{3}$ ) where $\mu$ and $\lambda$ are the Lamé constants.

\begin{tabular}{rrrrrrr}
\hline \hline$i$ & $j$ & $f$ & \multicolumn{1}{c}{$A$} & \multicolumn{1}{c}{$B$} & \multicolumn{1}{c}{$C$} & \multicolumn{1}{c}{$D$} \\
\hline 5 & 0 & -2 & 118 & 177 & 78 & 57 \\
4 & 0,1 & 1 & 1910 & 5229 & 5292 & 1959 \\
3 & 0,2 & -2 & 1180 & 5712 & 7221 & 2712 \\
& 1 & -4 & 310 & 2547 & 2691 & 192 \\
2 & 0,3 & 1 & 1850 & 6379 & 7572 & 2649 \\
& 1,2 & -3 & 130 & 2031 & 2308 & -129 \\
1 & 0,4 & -2 & 280 & 422 & 111 & 132 \\
& 1,3 & 2 & 950 & 3547 & 3531 & 1362 \\
& 2 & -6 & 230 & 1449 & 2822 & 1209 \\
0 & 0,5 & 1 & 14 & 25 & 36 & -15 \\
& 1,4 & -5 & 46 & 5 & -90 & -111 \\
& 2,3 & -5 & 26 & 103 & 522 & 219 \\
\hline \hline
\end{tabular}

$$
\mathbf{G}_{m}^{\Xi \odot}\left(x_{1}, x_{2}, x_{3}\right)=\frac{P_{m}^{\Xi}}{|\mathbf{x}|^{4 m+5}}\left(\begin{array}{c}
G_{m \|}^{\Xi \odot}\left(x_{1}, x_{2}, x_{3}\right) \\
G_{m \|}^{\Xi \odot}\left(x_{2}, x_{3}, x_{1}\right) \\
G_{m \|}^{\Xi \odot}\left(x_{3}, x_{1}, x_{2}\right) \\
G_{m \perp}^{\odot}\left(x_{2}, x_{3}, x_{1}\right) \\
G_{m \perp}^{\odot}\left(x_{1}, x_{3}, x_{2}\right) \\
G_{m \perp}^{\odot}\left(x_{1}, x_{2}, x_{3}\right)
\end{array}\right),
$$

where the two distinct functions are given the symbols $\|$ and $\perp$ to indicate plane and shear components. In general, the plane components represented by $G_{m \|}^{\Xi \odot}$ depend on whether they relate to stress $(\Xi \equiv \sigma)$ or strain $(\Xi \equiv \epsilon)$ but the shear components differ only through the constant $P_{m}^{\Xi}$.

The constants $P_{m}^{\Xi}$ are

$$
\begin{aligned}
& P_{m}^{\epsilon}=\frac{\epsilon_{0}}{m ! 4^{m+1} \pi \mu^{m}(2 \mu+\lambda)}, \\
& P_{m}^{\sigma}=2 \mu P_{m}^{\epsilon} .
\end{aligned}
$$

where $\epsilon_{0}$ is the uniform misfit strain in an unrelaxed QD using the convention that $\epsilon_{0}$ is negative for a compressively strained QD. The Green's functions for $m=0$ are

$$
G_{0 \|}^{\Xi \odot}\left(x_{1}, x_{2}, x_{3}\right)=-(2 \mu+3 \lambda)\left(|\mathbf{x}|^{2}-3 x_{1}^{2}\right),
$$

TABLE VI. The coefficients $M_{i j}^{\epsilon \odot}$ are of the form $f\left(A \mu^{3}+B \mu^{2} \lambda+C \mu \lambda^{2}\right.$ $+D \lambda^{3}$ ) where $\mu$ and $\lambda$ are the Lamé constants.

\begin{tabular}{|c|c|c|c|c|c|c|}
\hline$k$ & $i$ & $f$ & A & $B$ & $C$ & $D$ \\
\hline 4 & 0 & 3 & 38 & 37 & 8 & 117 \\
\hline 3 & 0,1 & -6 & 374 & 1221 & 1524 & 531 \\
\hline \multirow[t]{2}{*}{2} & 0,2 & 3 & 818 & 3687 & 4248 & 1677 \\
\hline & 1 & 8 & 176 & 1094 & 666 & -81 \\
\hline \multirow[t]{2}{*}{1} & 0,3 & -6 & 294 & 871 & 929 & 276 \\
\hline & 1,2 & 2 & 784 & 5566 & 5499 & 981 \\
\hline \multirow[t]{3}{*}{0} & 0,4 & 3 & 118 & 177 & 78 & 57 \\
\hline & 1,3 & -2 & 1042 & 3103 & 3312 & 1233 \\
\hline & 2 & 1 & 2054 & 10781 & 14004 & 5121 \\
\hline
\end{tabular}

\begin{tabular}{rrrrrrr}
\hline \hline$i$ & $j$ & $f$ & $A$ & $B$ & \multicolumn{1}{c}{$C$} & \multicolumn{1}{c}{$D$} \\
\hline 5 & 0 & -2 & -6 & 35 & 44 & 3 \\
4 & 0,1 & -1 & 1350 & 2477 & 2060 & 741 \\
3 & 0,2 & 2 & 1860 & 7336 & 7405 & 2373 \\
& 1 & 4 & 1350 & 5611 & 4975 & 1158 \\
2 & 0,3 & -1 & 1690 & 7307 & 11080 & 4371 \\
& 1,2 & 3 & 1170 & 3223 & 340 & -621 \\
1 & 0,4 & 2 & 80 & -74 & -25 & 273 \\
& 1,3 & -2 & 1270 & 5291 & 5755 & 1158 \\
& 2 & 6 & 150 & 197 & 380 & 621 \\
0 & 0,5 & 1 & 2 & -17 & 92 & 3 \\
& 1,4 & 5 & 14 & -11 & 122 & 39 \\
& 2,3 & -5 & 86 & 457 & 230 & 75 \\
\hline \hline
\end{tabular}

TABLE VII. The coefficients $N_{k i}^{\odot}$ are of the form $f\left(A \mu^{3}+B \mu^{2} \lambda+C \mu \lambda^{2}\right.$ $+D \lambda^{3}$ ) where $\mu$ and $\lambda$ are the Lamé constants.

Equations (19) and (20) combined with Eq. (16) with $m=0$ yield the well-known stress or strain Green's matrix for isotropic crystals. ${ }^{17}$ We note that the only difference between the stress and strain Green's matrices for the $m=0$ case is a factor of $2 \mu$ that appears in the constant given by Eq. (18). In other words, Eq. (19) is identical for both stress and strain for the isotropic case $m=0$. The $m>0$ terms approximate the cubic anisotropy of the crystal. We evaluate these for $m=1$ and $m=2$ only.

The Green's matrix for $m=1$ may be written as

$$
\begin{aligned}
& G_{1 \|}^{\Xi \odot}\left(x_{1}, x_{2}, x_{3}\right)=\sum_{i=0}^{3} \sum_{j=0}^{3-i} K_{i j}^{\Xi \odot} x_{1}^{2 i} x_{2}^{2 j} x_{3}^{6-2 i-2 j}, \\
& G_{1 \perp}^{\odot}\left(x_{1}, x_{2}, x_{3}\right)=x_{1} x_{2} \sum_{k=0}^{2} \sum_{i=0}^{2-k} L_{k i}^{\odot} x_{3}^{2 k} x_{1}^{2 i} x_{2}^{4-2 i-2 k},
\end{aligned}
$$

and are polynomials of order 6 . The constants $K^{\sigma \odot}, K^{\epsilon \odot}$, and $L^{\odot}$ are presented in Tables II-IV.

The functions forming the $m=2$ Green's tensor contain polynomials of order 10 ,

$$
\begin{aligned}
& G_{2 \|}^{\Xi \odot}\left(x_{1}, x_{2}, x_{3}\right)=\sum_{i=0}^{5} \sum_{j=0}^{5-i} M_{i j}^{\Xi \odot} x_{1}^{2 i} x_{2}^{2 j} x_{3}^{10-2 i-2 j}, \\
& G_{2 \perp}^{\odot}\left(x_{1}, x_{2}, x_{3}\right)=x_{1} x_{2} \sum_{k=0}^{4} \sum_{i=0}^{4-k} N_{k i}^{\odot} x_{3}^{2 k} x_{1}^{2 i} x_{2}^{8-2 i-2 k},
\end{aligned}
$$

where the constants $M^{\sigma \odot}, M^{\epsilon \odot}$, and $N^{\odot}$ are presented in Tables V-VII. Files containing the coefficients presented in the tables can be obtained by emailing the author.

The full Green's matrix for either stress or strain, to second order in $\Delta$, can therefore be obtained from Eqs. (16)-(24). The stress or strain matrix for a QD is obtained by integrating the stress or strain Green's matrix over the volume of the dot. Thus, for stress,

$$
\boldsymbol{\sigma}^{\odot}\left(x_{1}, x_{2}, x_{3}\right)=\int_{V} \mathbf{G}^{\sigma \odot}\left(x_{1}^{\prime}, x_{2}^{\prime}, x_{3}^{\prime}\right) d V\left(x_{1}^{\odot}, x_{2}^{\odot}, x_{3}^{\odot}\right),
$$

where $x_{i}^{\prime}=x_{i}-x_{i}^{\odot}$ are Cartesian coordinates where $x_{i}^{\odot}$ refers to points within the volume of the QD and $V$ therefore represents the volume of the dot. 
TABLE VIII. The coefficients $J_{i j}^{\odot}$ for the calculation of the QD hydrostatic strain are of the form $f\left(A \mu^{2}+B \mu \lambda+C \lambda^{2}\right)$ where $\mu$ and $\lambda$ are the Lamé constants.

\begin{tabular}{crrrr}
\hline \hline$i j$ & \multicolumn{1}{c}{$f$} & \multicolumn{1}{c}{$A$} & \multicolumn{1}{c}{$B$} & \multicolumn{1}{c}{$C$} \\
\hline $40,00,04$ & 1 & 4 & -26 & 24 \\
$30,31,10,13,01,03$ & -1 & 284 & 644 & 399 \\
$20,22,02$ & 6 & 114 & 319 & 174 \\
$21,11,12$ & 6 & 108 & 268 & 3 \\
\hline \hline
\end{tabular}

The analytic expressions for the components of the Green's matrix can be extremely useful. For example, the hydrostatic strain is defined as $\epsilon_{h}=\epsilon_{11}+\epsilon_{22}+\epsilon_{33}$ and so the $m$ th hydrostatic strain Green's function, $G_{m}^{h \odot}$, is easily obtained. We find that these Green's functions are of a particularly simple form

$$
\begin{aligned}
G_{0}^{h \odot}= & 0, \\
G_{1}^{h \odot}= & \frac{12 \mu(2 \mu+3 \lambda) P_{1}^{\epsilon}}{|\mathbf{x}|^{7}}\left\{x_{1}^{4}+x_{2}^{4}+x_{3}^{4}-3\left(x_{1}^{2} x_{2}^{2}+x_{2}^{2} x_{3}^{2}\right.\right. \\
& \left.\left.+x_{3}^{2} x_{1}^{2}\right)\right\} \\
G_{2}^{h \odot}= & \frac{4 \mu P_{2}^{\epsilon}}{|\mathbf{x}|^{11}} \sum_{i=0}^{4} \sum_{j=0}^{4-i} J_{i j}^{\odot} x_{1}^{2 i} x_{2}^{2 j} x_{3}^{8-2 i-2 j},
\end{aligned}
$$

where the constants $J^{\odot}$ are listed in Table VIII. The hydrostatic strain shifts the conduction-band- and average valenceband-edge energies ${ }^{18,36}$ and is an important quantity in electronic structure analysis. Equation (26) indicates that the Green's function is zero in the isotropic approximation and so the direct integration over the volume of a QD will also yield a zero result. It is always necessary to add the initial conditions to obtain the correct hydrostatic strain so that $4 \epsilon_{0} \mu /(\lambda+2 \mu)$ is added to the volume integrals obtained from Eqs. (27) and (28) for a field point inside the QD. Equation (27) was presented by Faux and Pearson. ${ }^{23}$

The stress or strain can therefore be computed for any QD shape by computing the Green's matrix through Eqs. (13)-(24) and then integrating over the volume of the dot. The principle of superposition allows the strain in systems containing large numbers of QDs to be determined and composition variation within the QD is also easily catered for by making $\epsilon_{0}$ a function of position and including it inside the integral.

These polynomial functions constitute very simple expressions which are straightforward to compute. No numerical computation is required to generate the Green's tensors

TABLE IX. The coefficients $K_{i}^{\sigma \Theta}$ for a quantum wire are of the form $f\left(A \mu^{2}+B \mu \lambda+C \lambda^{2}\right)$ where $\mu$ and $\lambda$ are the Lamé constants.

\begin{tabular}{rrrrr}
\hline \hline$i$ & $f$ & $A$ & $B$ & $C$ \\
\hline 3 & 2 & 14 & 23 & 15 \\
2 & -2 & 58 & 157 & 93 \\
1 & 6 & 6 & 19 & 11 \\
0 & -6 & 2 & 1 & 1 \\
\hline \hline
\end{tabular}

TABLE X. The coefficients $K_{i}^{\epsilon \Theta}$ for a quantum wire are of the form $f\left(A \mu^{2}+B \mu \lambda+C \lambda^{2}\right)$ where $\mu$ and $\lambda$ are the Lamé constants.

\begin{tabular}{rrrrr}
\hline \hline$i$ & $f$ & $A$ & $B$ & $C$ \\
\hline 3 & 6 & 2 & 1 & 1 \\
2 & -6 & 22 & 51 & 23 \\
1 & 2 & 26 & 93 & 69 \\
0 & 2 & 2 & 9 & 3 \\
\hline \hline
\end{tabular}

but the truncated series is not exact. Nevertheless, we show in Sec. III that they form an excellent approximation to the exact results for cubic anisotropy.

\section{B. Quantum wires}

One of the significant advantages of developing analytic forms for the QD Green's tensors is that they may be integrated with respect to one coordinate, $x_{2}$ say, to obtain immediately the Green's matrix for a line source. Thus,

$$
\mathbf{G}^{\Xi \Theta}\left(x_{1}, x_{3}\right)=\int_{-\infty}^{\infty} \mathbf{G}^{\Xi \odot}\left(x_{1}, x_{2}, x_{3}\right) d x_{2} .
$$

where the $\Theta$ symbol is used to indicate a quantum wire with a cross section in the $\left(x_{1}, x_{3}\right)$ plane. The stress or strain Green's matrix for a line source, if integrated over the crosssectional area of a QWI, yields the stress or strain, respectively, at a position in the $\left(x_{1}, x_{3}\right)$ plane due to a strained QWI. The Green's tensors for the QWI can be expressed as

$$
\mathbf{G}_{m}^{\Xi \Theta}\left(x_{1}, x_{3}\right)=\frac{P_{m}^{\Xi}}{|\mathbf{X}|^{4 m+4}}\left(\begin{array}{c}
G_{m \|}^{\Xi \Theta}\left(x_{1}, x_{3}\right) \\
0 \\
G_{m \|}^{\Xi \Theta}\left(x_{3}, x_{1}\right) \\
0 \\
G_{m \perp}^{\Theta}\left(x_{1}, x_{3}\right) \\
0
\end{array}\right),
$$

where, for the QWI, $|\mathbf{x}|^{2}=x_{1}^{2}+x_{3}^{2}$. The QWI Green's tensor for $m=0$ may be written as

$$
\begin{aligned}
& G_{0 \|}^{\Xi \Theta}\left(x_{1}, x_{3}\right)=2(2 \mu+3 \lambda)\left(x_{1}^{2}-x_{3}^{2}\right), \\
& G_{0 \perp}^{\Theta}\left(x_{1}, x_{3}\right)=4(2 \mu+3 \lambda) x_{1} x_{3} .
\end{aligned}
$$

Equations (31) and (32) combined with Eq. (30) with $n=0$ yield the well-known stress or strain Green's matrix for isotropic crystals. $^{14}$

The QWI Green's tensor for $m=1$ may be written as

$$
G_{1 \|}^{\Xi \Theta}\left(x_{1}, x_{3}\right)=\sum_{i=0}^{3} K_{i}^{\Xi \Theta} x_{1}^{2 i} x_{3}^{6-2 i},
$$

TABLE XI. The coefficients $L_{k}^{\Theta}$ for a quantum wire are of the form $f\left(A \mu^{2}+B \mu \lambda+C \lambda^{2}\right)$ where $\mu$ and $\lambda$ are the Lamé constants.

\begin{tabular}{ccccc}
\hline \hline$k$ & $f$ & $A$ & $B$ & $C$ \\
\hline 2,0 & 4 & 14 & 23 & 15 \\
1 & -8 & 10 & 37 & 21 \\
\hline \hline
\end{tabular}


TABLE XII. The coefficients $M_{i}^{\sigma \Theta}$ for a quantum wire are of the form $f\left(A \mu^{3}+B \mu^{2} \lambda+C \mu \lambda^{2}+D \lambda^{3}\right)$ where $\mu$ and $\lambda$ are the Lamé constants.

\begin{tabular}{rrrrrr}
\hline \hline$i$ & $f$ & $A$ & \multicolumn{1}{c}{$B$} & \multicolumn{1}{c}{$C$} & \multicolumn{1}{c}{$D$} \\
\hline 5 & -2 & 38 & 37 & -24 & 21 \\
4 & 6 & 234 & 531 & 536 & 243 \\
3 & -4 & 470 & 2445 & 3048 & 1005 \\
2 & 4 & 358 & 1157 & 2512 & 501 \\
1 & -6 & 58 & 43 & 24 & 27 \\
0 & -2 & 10 & 3 & 24 & 3 \\
\hline \hline
\end{tabular}

$$
G_{1 \perp}^{\Theta}\left(x_{1}, x_{3}\right)=x_{1} x_{3} \sum_{k=0}^{2} L_{k}^{\Theta} x_{3}^{2 k} x_{1}^{4-2 k}
$$

The constants $K^{\sigma \Theta}, K^{\epsilon \Theta}$, and $L^{\Theta}$ are presented in Tables IX-XI.

The functions forming the $m=2$ QWI Green's tensor are polynomials of order 10 ,

$$
\begin{aligned}
& G_{2 \|}^{\Xi \Theta}\left(x_{1}, x_{3}\right)=\sum_{i=0}^{5} M_{i}^{\Xi \Theta} x_{1}^{2 i} x_{3}^{10-2 i}, \\
& G_{2 \perp}^{\Theta}\left(x_{1}, x_{3}\right)=x_{1} x_{3} \sum_{k=0}^{4} N_{k}^{\Theta} x_{3}^{2 k} x_{1}^{8-2 k},
\end{aligned}
$$

where the constants $M^{\sigma \Theta}, M^{\epsilon \Theta}$, and $N^{\Theta}$ are listed in Tables XII-XIV.

The zeroth-order hydrostatic strain, as for the QD, is zero. The hydrostatic Green's function $G_{1}^{h \Theta}\left(x_{1}, x_{3}\right)$, defined as $G_{1 \|}^{\epsilon \Theta}\left(x_{1}, x_{3}\right)+G_{1 \|}^{\epsilon \Theta}\left(x_{3}, x_{1}\right)$ as the first-order correction for a QWI, is found to be

$$
G_{1}^{h \Theta}\left(x_{1}, x_{3}\right)=\frac{8 \mu P_{1}^{\epsilon}}{|\mathbf{x}|^{6}} \sum_{i=0}^{2} H_{i}^{\Theta} x_{1}^{2 i} x_{3}^{4-2 i},
$$

where $H_{0}^{\Theta}=H_{2}^{\Theta}=(2 \mu+3 \lambda)$ and $H_{1}^{\Theta}=-6(2 \mu+3 \lambda)$. We note the especially simple form of this expression. The secondorder hydrostatic Green's function $G_{2}^{h \Theta}\left(x_{1}, x_{3}\right)$ is

$$
G_{2}^{h \Theta}\left(x_{1}, x_{3}\right)=\frac{16 \mu P_{2}^{\epsilon}}{|\mathbf{x}|^{10}} \sum_{i=0}^{4} J_{i}^{\Theta} x_{1}^{2 i} x_{3}^{8-2 i},
$$

where

$$
\begin{aligned}
& J_{0}^{\Theta}=J_{4}^{\Theta}=\left(-2 \mu^{2}-9 \mu \lambda+3 \lambda^{2}\right), \\
& J_{1}^{\Theta}=J_{3}^{\Theta}=-4\left(10 \mu^{2}+21 \mu \lambda+21 \lambda^{2}\right), \\
& J_{2}^{\Theta}=10\left(18 \mu^{2}+49 \mu \lambda+21 \lambda^{2}\right) .
\end{aligned}
$$

These Green's functions enable the hydrostatic strain in and around a QWI to be computed simply and quickly.

\section{RESULTS}

We undertake a careful assessment of the accuracy of the Green's tensors produced from the formalism presented in Sec. II. In earlier work, ${ }^{23}$ the strain component $\epsilon_{11}$ was calculated for a spherical QD, for the region outside the QD in the (100) direction, and compared to the approximate Fourier
TABLE XIII. The coefficients $M_{i}^{\epsilon \Theta}$ for a quantum wire are of the form $f\left(A \mu^{3}+B \mu^{2} \lambda+C \mu \lambda^{2}+D \lambda^{3}\right)$ where $\mu$ and $\lambda$ are the Lamé constants.

\begin{tabular}{rrrrrr}
\hline \hline$i$ & $f$ & $A$ & \multicolumn{1}{c}{$B$} & \multicolumn{1}{c}{$C$} & \multicolumn{1}{c}{$D$} \\
\hline 5 & -2 & 10 & 3 & 24 & 3 \\
4 & -6 & 90 & 11 & 48 & 51 \\
3 & 20 & 174 & 641 & 584 & 177 \\
2 & -4 & 310 & 1581 & 2416 & 885 \\
1 & -6 & 22 & 237 & 168 & 51 \\
0 & -6 & 2 & 23 & -16 & -1 \\
\hline \hline
\end{tabular}

approach of Andreev et al. ${ }^{33}$ A very good agreement was obtained. Here, we use the Pan approach as the standard. ${ }^{24,28-30}$ In principle, Pan's theory provides an exact Green's tensor for crystals with any form of anisotropy and so is extremely powerful. However, as usual with a more general formalism, implementation is less straightforward. Pan's approach requires significant computation, including finding the numerical solution to an eighth-order polynomial equation and the numerical differentiation of the displacement Green's tensor to obtain the stress or strain Green's tensor. For the purposes of direct comparison, we use the volume integral form of the Pan's Green's tensor. For simplicity, we refer to the Green's approach presented in Sec. II as Faux-Christmas (FC).

First, the numerical values of the strain FC Green's tensor for a QD are compared to the Pan equivalent. Direct comparison of the Green's functions is preferable to a comparison of the strain for a specific QD as it avoids the numerical difficulties associated with integration over a volume of the QD. Material parameters for GaAs are used (see Table I) and $\epsilon_{0}=0.01$. The source is at the origin. The Green's tensor is calculated for each of the three cases; first, the isotropic case (where $\mu^{\prime}=0$ and values of $\mu$ and $\lambda$ are used), second the FC polynomial solutions obtained from Eq. (16) with the substitutions given by Eqs. (17)-(24), and finally the Pan approach. In the implementation of the Pan theory, the strain is coupled with the piezoelectric field. The computation is much more stable if the full piezoelectric constants are included. However, for GaAs, piezoelectric effects are very small and we have determined that they have a negligible effect on the current results.

Figure 1 presents the absolute values of $G_{\|}^{\epsilon \odot}\left(x_{1}, x_{2}, x_{3}\right)$ and $G_{\perp}^{\epsilon \odot}\left(x_{1}, x_{2}, x_{3}\right)$ plotted as a function of distance $r$ from the source (origin) in the (100) and (111) directions, respectively. We have chosen to represent the distance in units of $\mathrm{nm}$ which leaves the Green's functions in units of $\mathrm{nm}^{-3}$. On a log-log plot the results are straight lines, as expected, with a gradient of -3 . In all cases, the Green's tensor is of the form $\epsilon_{0} \mathbf{A} / r^{3}$ where $\mathbf{A}$ is a dimensionless tensor independent

TABLE XIV. The coefficients $N_{k}^{\Theta}$ for a quantum wire are of the form $f\left(A \mu^{3}+B \mu^{2} \lambda+C \mu \lambda^{2}+D \lambda^{3}\right)$ where $\mu$ and $\lambda$ are the Lamé constants.

\begin{tabular}{crrrrr}
\hline \hline$k$ & $f$ & $A$ & $B$ & $C$ & \multicolumn{1}{c}{$D$} \\
\hline 4,0 & 4 & 38 & 37 & -24 & 21 \\
3,1 & -16 & 98 & 247 & 280 & 111 \\
2 & 24 & 70 & 405 & 488 & 165 \\
\hline \hline
\end{tabular}




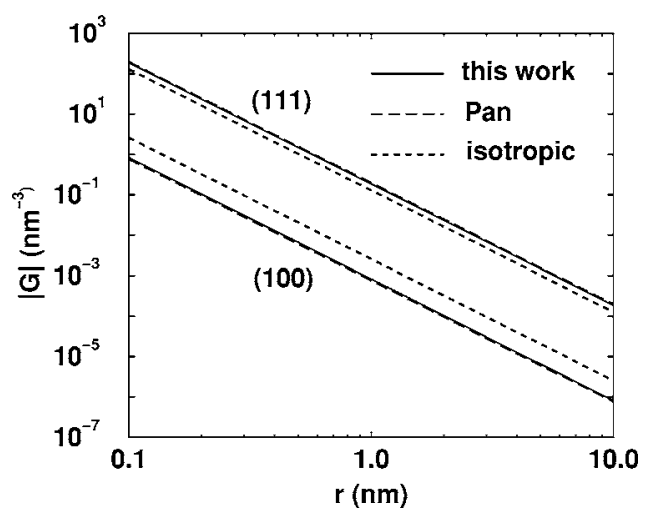

FIG. 1. The absolute values of $G_{\|}^{\epsilon \odot}\left(x_{1}, x_{2}, x_{3}\right)$ in the (100) direction and $G_{\perp}^{\epsilon \odot}\left(x_{1}, x_{2}, x_{3}\right)$ in the (111) direction are plotted as a function of distance from the source (origin) $r$. The results for $G_{\perp}^{\epsilon \odot}\left(x_{1}, x_{2}, x_{3}\right)$ have been displaced vertically by two decades for clarity.

of $r$ but a function of the angles $\theta$ and $\phi$. Indeed, the substantial theoretical effort involved in deriving the Green's tensors for anisotropic elasticity essentially reduces to finding the form for $\mathbf{A}$. The values of the tensor components of $\mathbf{A}$ for the isotropic, FC, and Pan approaches are presented in Table XV.

In all cases, the FC results are much closer to the Pan values than for the standard isotropic approach. For example, in the (100) direction, the FC approach makes up about $96 \%$ of the difference between the isotropic value and the accurate Pan result. Even in the worse case, the FC value contributes more than $80 \%$ of the difference (except in one case where all methods give similar values) and therefore constitutes a significant improvement in accuracy with a minimum of additional computation. Dederichs and Leibfried ${ }^{37}$ used a perturbation theory to approximate the strain field due to a point source in crystals with cubic anisotropy. They presented "best" isotropic elastic stiffnesses to be used for strain evaluation for crystals with cubic anisotropy. We found, however, that their expressions for the effective elastic stiffnesses produced little improvement on the standard isotropic theory.

A stringent test of the FC approximation is to determine the component of the strain in the direction normal to the plane of a quantum well, call this the $z^{*}$ direction, for a quantum well orientated such that the $z^{*}$ axis points along (001), (110), or (111). Analytical solutions are known for the constant strain within the well and are ${ }^{38}$

TABLE XV. The value of the components of $\mathbf{A}$ in the expression $\epsilon_{0} \mathbf{A} / r^{3}$ describing the form of the strain Green's components for the Pan and Yang (Ref. 24), Faux-Christmas (FC), and isotropic approaches for a point source of strain in GaAs.

\begin{tabular}{clcrr}
\hline \hline Direction & Green's function & Pan & \multicolumn{1}{c}{ FC } & Isotropic \\
\hline \multirow{2}{*}{100} & $A_{11}$ & 0.0757 & 0.0830 & 0.2583 \\
& $A_{22}=A_{33}$ & -0.0993 & -0.1019 & -0.1292 \\
& $A_{12}=A_{13}=A_{23}$ & 0.000 & 0.0000 & 0.0000 \\
110 & $A_{11}=A_{22}$ & 0.134 & 0.1217 & 0.0646 \\
& $A_{33}$ & -0.199 & -0.1947 & -0.1292 \\
& $A_{12}$ & 0.193 & 0.1919 & 0.1937 \\
& $A_{13}=A_{23}$ & 0.000 & 0.0000 & 0.0000 \\
111 & $A_{11}=A_{22}=A_{33}$ & 0.0464 & 0.0392 & 0.0000 \\
& $A_{12}=A_{13}=A_{23}$ & 0.198 & 0.1863 & 0.1292 \\
\hline \hline
\end{tabular}

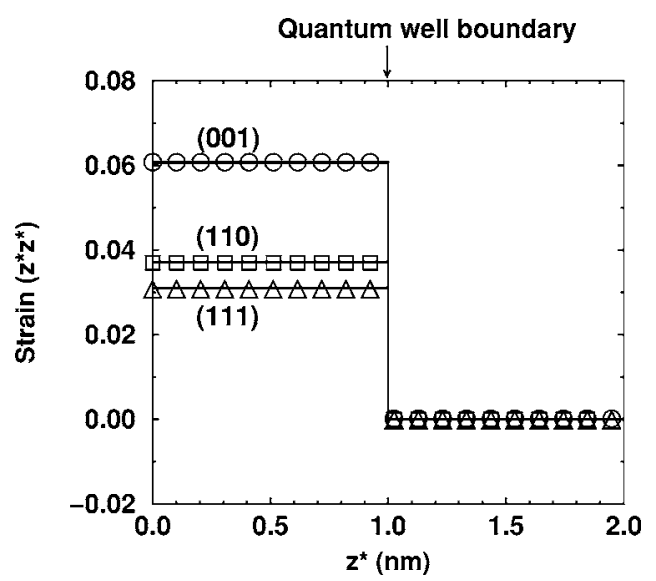

FIG. 2. The strain component $\epsilon_{z^{*}} z^{*}$ in the direction normal to the plane of a quantum well is presented for the (001), (110), and (111) directions as a function of distance in the $z^{*}$ direction for the Faux-Christmas approximation (symbols) and for the exact analytic formulas (solid lines). The quantum well is $2 \mathrm{~nm}$ thick with a misfit strain of -0.067 and centerd on the origin. $\mathrm{GaAs}$ elastic constants are assumed.

$$
\begin{aligned}
& \frac{\epsilon_{z^{*} z^{*}}}{\epsilon_{0}}=\frac{-2 \lambda}{C_{11}}, \quad, \\
&=\frac{2 \mu-3 \lambda-C_{11}}{2 \mu+\lambda+C_{11}}, \\
&=2 \frac{2 \mu-2 \lambda-C_{11}}{4 \mu+2 \lambda+C_{11}},
\end{aligned}
$$

where Eq. (42) also corresponds to the isotropic result. The strain component $\epsilon_{z^{*} z^{*}}$ in the direction normal to the plane of a quantum well is presented in Fig. 2 for the (001), (110), and (111) directions as a function of distance in the $z^{*}$ direction using Eqs. (42)-(44) and the FC method. The FC results are computed by numerical integration of the Green's tensor as described in Sec. II as if the quantum well were a cuboidal QD with dimensions of $200 \times 200 \times 2 \mathrm{~nm}^{3}$. GaAs elastic constants are assumed throughout and the misfit strain is taken to be -0.067 . The FC results are in excellent agreement with the exact results for all orientations of the well.

Finally we examine the hydrostatic strain for a cuboidal QD with dimensions of $18 \times 18 \times 6 \mathrm{~nm}^{3}$ with growth direction along the $z^{*}$ axis. The chosen dot size is consistent with a range of QDs including the truncated pyramid characteristic of InAs/GaAs systems (for example, see Ref. 39), the lens or cylinder, and so these results illustrate the general trends to be found in a range of QD systems. Elastic constants for GaAs are again used for all materials and the misfit strain is taken to be -0.067 .

We focus on three cases with the growth direction along (001), (110), and (111). With the $z^{*}$ axis orientated along (110), the $x^{*}$ and $y^{*}$ axes are taken to be along (110) and (001), respectively, and when the $z^{*}$ axis is orientated along (111) the $x^{*}$ and $y^{*}$ axes are in the $(1 \overline{1} 0)$ and $(11 \overline{2})$ directions, respectively. The hydrostatic strain is calculated using Eqs. (26)-(28). Figure 3 presents $\epsilon_{h}$ as a function of distance along the $z^{*}$ axis through the center of the dot along (001), (110), and (111). Inside the QD, $\epsilon_{h}$ is only a weak function of 


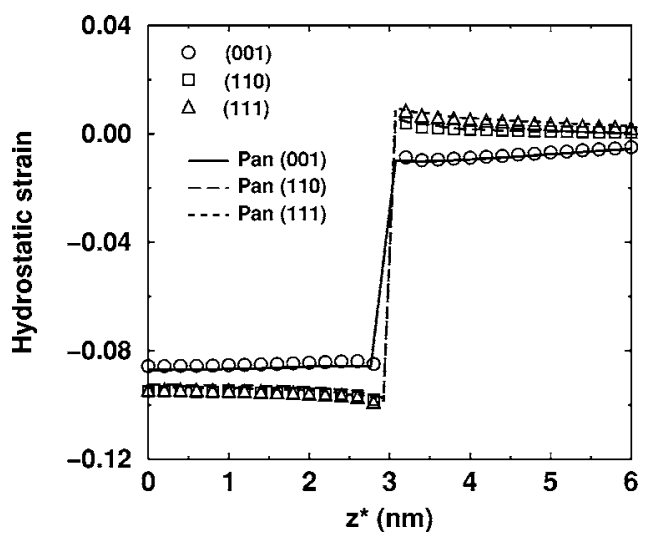

FIG. 3. The hydrostatic strain for a cuboidal InAs/GaAs QD with dimensions of $18 \times 18 \times 6 \mathrm{~nm}^{3}$ with growth direction along the $z^{*}$ axis is presented and compared to the results of the Pan method (Ref. 24). GaAs elastic constants are assumed with a misfit strain of -0.067 .

position and a weak function of growth direction. The hydrostatic strain at the center of the QD is about -0.095 . In the (001) direction, the hydrostatic strain decreases in magnitude towards the edge of the dot reaching -0.086 at the boundary whereas, in the other directions, its value is approximately constant. Outside the QD, $\epsilon_{h}$ is zero in the isotropic approximation but Fig. 3 demonstrates clearly that the cubic anisotropy of the elastic constants leads to nonzero hydrostatic strain. $\epsilon_{h}$ is positive with a maximum value of 0.007 for a QD grown on a (111) surface and about -0.007 for growth along the (001) axis. So, for example, the change in hydrostatic strain across the boundary of the QD would be 0.092 using the isotropic model, but only 0.079 if the cubic anisotropy of the elastic constants is taken into account for a QD grown on a (100) surface. The hydrostatic strain is nonzero outside the dot over distances of the order of the dimension of the dot.

The real-space Green's tensor expansion allows the straightforward determination of either the stress or strain fields due to a uniformly strained quantum dot buried in an anisotropic medium through a volume integral of a simple polynomial function. Other approaches, including the PanYang model, are much more complex to implement as several numerical stages are required. Thus, the computation of the strain at a field point, for example, is about 50 times faster using the Green's tensor expansion than the Pan-Yang method. One example of a result that can only be computed accurately using the current method is the estimation of the energy of interaction of two point defects. ${ }^{31}$ To illustrate, we have estimated the strain interaction energy between a $\mathrm{Si}$ interstitial and a $\mathrm{C}$ substitutional atom in a $\mathrm{Si}$ crystal. The interaction energy is presented in Fig. 4 as a function of the defect separation. We found that, if the line joining the two defects is in the (100) or (111) direction, the energy is positive or negative, indicating a mutually repulsive or attractive force, respectively. The energy is proportional to the inverse third power of the distance between the two defects in both cases. These results agree with the results of Masumura and Sines, ${ }^{40}$ Schaefer and Kronmüller, ${ }^{41}$ and Shneck et al. ${ }^{42}$ for cubic systems. Even if computation time was not a problem, we found that the numerical computation necessary using the

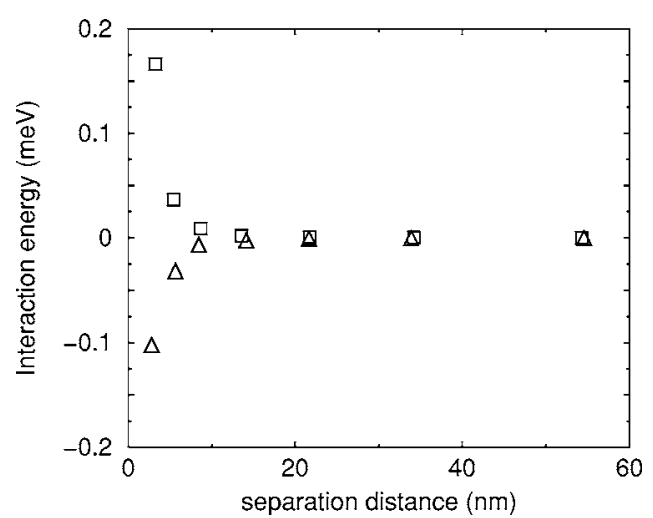

FIG. 4. Strain interaction energy between a Si interstitial and a $\mathrm{C}$ substitutional atom in a $\mathrm{Si}$ crystal, as a function of distance between the point defects. The line joining the defects lies along the (100) direction (square symbols) and along the (111) direction (triangles).

Pan-Yang approach leads to energies that are only accurate to three or four significant figures. Normally, this would be perfectly acceptable, but when two similar energies are subtracted, the numerical errors become significant.

\section{CONCLUSIONS}

We have presented the real-space stress and strain Green's tensors enabling the calculation of strain distributions including the cubic anisotropy of the elastic constants as a polynomial series for buried quantum dots and quantum wires. The zeroth term of the series is equivalent to the isotropic result. We find that the Green's tensors presented in Sec. II are very straightforward to implement and compute considerably faster than alternative approaches. The present results are compared to alternative solutions that include the cubic anisotropy of the elastic constants for a GaAs QD and very good accuracy is demonstrated when the first- and second-order terms of the Green's tensor series are included. The Green's functions for the hydrostatic strain are shown to be of a very simple form for both the QD and QWI. QD or QWI arrays or nanostructures with compositional variation are trivial extensions of the method. The Green's function for the hydrostatic strain is shown to be of a particularly simple form and it is shown that the presence of cubic anisotropy introduces a weak spatial variation of hydrostatic strain inside the QD and nonzero hydrostatic strain outside the dot.

${ }^{1}$ Y. Arakawa and H. Sakaki, Appl. Phys. Lett. 40, 939 (1982).

${ }^{2}$ D. Bimberg, M. Grundmann, and N. N. Ledentsov, Quantum Dot Heterostructures (Wiley, New York, 1999).

${ }^{3}$ M. Tadić, F. M. Peeters, and K. L. Janssens, Phys. Rev. B 65, 5819 (2002).

${ }^{4}$ M. Tadić, F. M. Peeters, and K. L. Janssens, Phys. Rev. B 65, 165333 (2002).

${ }^{5}$ H. Heidemeyer, D. U. Denker, C. Müller, and G. Schmidt, Phys. Rev. Lett. 91, 196103 (2003).

${ }^{6}$ M. Grundmann, O. Stier, and D. Bimberg, Phys. Rev. B 52, 11969 (1995).

${ }^{7}$ M. A. Migliorato, A. G. Cullis, M. Fearn, and J. H. Jefferson, Phys. Rev. B 41, 3930 (2002).

${ }^{8}$ M. A. Makeev and A. Madhukar, Phys. Rev. B 67, 073201 (2003).

${ }^{9}$ A. J. Williamson, L. W. Wang, and Z. Zunger, Phys. Rev. B 62, 2963 (2000).

${ }^{10}$ Q. X. Pei, C. Lu, and Y. Y. Wang, J. Appl. Phys. 93, 1487 (2003).

${ }^{11}$ A. D. Andreev and E. P. O’Reilly, Phys. Rev. B 92, 15851 (2000).

${ }^{12}$ D. A. Faux, J. R. Downes, and E. P. O’Reilly, J. Appl. Phys. 82, 3754 
(1997).

${ }^{13}$ G. S. Pearson and D. A. Faux, J. Appl. Phys. 88, 730 (2000).

${ }^{14}$ D. A. Faux, J. R. Downes, and E. P. O'Reilly, J. Appl. Phys. 80, 2515 (1996).

${ }^{15}$ F. Glas, Phys. Status Solidi B 237, 599 (2003).

${ }^{16}$ J. R. Downes, D. A. Faux, and E. P. O'Reilly, J. Appl. Phys. 81, 6700 (1997).

${ }^{17}$ J. H. Davies, J. Appl. Phys. 84, 358 (1998)

${ }^{18}$ J. H. Davies, Appl. Phys. Lett. 75, 4142 (1999).

${ }^{19}$ T. J. Gosling, Philos. Mag. A 73, 11 (1996).

${ }^{20}$ N. A. Gippius and S. G. Tikhodeev, J. Exp. Theor. Phys. 88, 1045 (1999).

${ }^{21}$ V. Holý, G. Springholz, M. Pinczolits, and G. Bauer, Phys. Rev. Lett. 83, 356 (1999)

${ }^{22}$ M. Pinczolits, G. Springholz, and G. Bauer, Phys. Rev. B 60, 11524 (1999).

${ }^{23}$ D. A. Faux and G. S. Pearson, Phys. Rev. B 62, R4798 (2000).

${ }^{24}$ E. Pan and B. Yang, J. Appl. Phys. 90, 6190 (2001).

${ }^{25}$ J. M. Burgers, Proc. K. Ned. Akad. Wet. 42, 378 (1939).

${ }^{26}$ D. M. Barnett, Phys. Status Solidi B 49, 741 (1972).

${ }^{27}$ T. Mura and N. Kinoshita, Phys. Status Solidi B 47, 607 (1971).

${ }^{28}$ E. Pan and F. Tonon, Int. J. Solids Struct. 37, 943 (2000).
${ }^{29}$ E. Pan, J. Appl. Phys. 91, 3785 (2002)

${ }^{30}$ E. Pan, J. Appl. Phys. 91, 6379 (2002).

${ }^{31}$ N. Cowern, U. M. E. Christmas, J. Benson, and D. A. Faux (unpublished).

${ }^{32}$ I. M. Lifshits and L. N. Rosentsveig, Zh. Eksp. Teor. Fiz. 17, 9 (1947).

${ }^{33}$ A. D. Andreev, J. R. Downes, D. A. Faux, and E. P. O'Reilly, J. Appl. Phys. 86, 297 (1999).

${ }^{34}$ S. W. Ellaway and D. A. Faux, J. Appl. Phys. 92, 3027 (2002).

${ }^{35}$ B. W. Char, K. O. Geddes, G. H. Gonnet, B. L. Leong, M. B. Monagan, and S. M. Watt, Maple Language Reference Manual: Maple V: The Future of Mathematics (Springer, New York, 1991).

${ }^{36}$ M. P. C. M. Krijn, Semicond. Sci. Technol. 6, 27 (1991).

${ }^{37}$ P. H. Dederichs and G. Leibfried, Phys. Rev. 188, 1175 (1969).

${ }^{38}$ L. De Caro and L. Tapfer, Phys. Rev. B 48, 2298 (1993).

${ }^{39}$ P. W. Fry et al., Phys. Rev. Lett. 84, 733 (2000).

${ }^{40}$ R. A. Masumura and G. Sines, J. Appl. Phys. 41, 3930 (1970).

${ }^{41}$ H. E. Schaefer and H. Kronmüller, Phys. Status Solidi B 67, 63 (1975).

${ }^{42}$ R. Shneck, R. Alter, A. Brokman, and M. P. Dariel, Philos. Mag. A 65, 797 (1992)

${ }^{43}$ M. Levinshtein, S. Rumyantsev, and M. Shur, Handbook Series on Semiconductor Parameters (World Scientific, Singapore, 1996). 